\title{
Le développement durable, du global au local. Une analyse des outils d'évaluation des acteurs publics locaux
}

\author{
Aurélien Boutaud $^{\mathrm{a}}$, Christian Brodhag ${ }^{\mathrm{b}}$ \\ a Sciences et génie de l'environnement, Cabinet Terr(e)itoires, 353 chemin de la Fruitière de Perroix, 74290 Talloires, France \\ b Sciences et génie de l'environnement, Centre SITE, École nationale supérieure des mines de Saint-Étienne, 158 cours Fauriel, \\ 42023 Saint-Étienne cedex 2, France
}

\begin{abstract}
Cette nouvelle contribution dans NSS sur le développement durable questionne l'instrumentation générée au sein des projets territoriaux, en particulier les systèmes d'indicateurs. Les auteurs y développent une analyse critique des relations entre les niveaux d'organisation concernés par les enjeux du développement durable et les indicateurs qui s'y réfèrent. Deux commentaires insistent sur la question de la finalité de ces systèmes en les reliant aux informations attendues par les décideurs publics. C'est bien cette relation concrète à l'intentionnalité et à la conception de projets qui fait de la mise en oeuvre du développement durable un acte politique.
\end{abstract}

La Rédaction

\section{Mots-clés :}

développement durable ; évaluation local/global ;

Résumé - Les outils d'évaluation du développement durable élaborés par les acteurs publics locaux sont susceptibles de nous informer très précisément sur le contenu que ces acteurs donnent à ce concept ambigu - une information particulièrement importante eu égard au rôle central des collectivités locales dans le processus de mise en œuvre du développement durable. Cette analyse nous montre que le développement durable est avant tout présenté comme un vecteur de consensus, ce qui se traduit par une approche sous la forme du triptyque «économie-social-environnement», dont chaque branche doit être traitée sur un pied d'égalité. Or, cette approche locale est discutable si on la resitue dans son contexte global. En particulier, la question se pose de savoir si, pour résoudre une problématique mondiale qui se caractérise précisément par de forts déséquilibres (sociaux, écologiques, etc.), la meilleure solution consiste à adopter une approche équilibrée du concept au niveau local. Ne risque-t-on pas d'entretenir de ce fait les déséquilibres globaux?

\section{Keywords:}

sustainable development; local sustainability; evaluation tools

\begin{abstract}
Sustainable development - from global to local scales. An analysis of evaluation tools developed by local public actors. Many evaluation tools have appeared in recent years in France and Switzerland in the field of sustainable development (SD). Most have been developed by local authorities. One major interest of these tools is that they enable us to precisely identify the perceptions of SD which local authorities tend to make their own. The analysis we made of these tools reveals a clear tendency for local authorities to consider SD as a consensual concept with which to tackle local policies or projects through a three dimensional approach (the so-called triptych approach based on ecological, economic and social dimensions). It is generally assumed that these three dimensions should be addressed in a strictly balanced manner, i.e. with each being accorded equal importance. Since such a "balanced" approach to SD seems to be increasingly popular at the local level, it is important to re-question it and set it in a broader and more global context. One question in particular needs to be explored: while most SD concerns at the global level seem to have their sources in growing social and/or ecological disparities, is it judicious to tackle the issue of SD at the local level through this "balanced" approach? Would it not be more relevant for local actors to prioritise those dimensions (social, ecological or economic) in which they tend to have the worst performances in the global context?
\end{abstract}

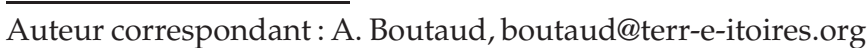


L'histoire du développement durable est indéniablement marquée par la confrontation entre deux formes d'intérêts potentiellement contradictoires : d'une part, la nécessité de préserver l'environnement mondial ; d'autre part, la volonté de maintenir une dynamique de développement socioéconomique à l'échelle de la planète ${ }^{1}$. Mais, s'il apparaît assez clairement que la formulation de ce concept est le fruit d'un processus de négociation de type coopératif - cherchant à concilier des points de vue et des intérêts divergents dans les domaines de l'environnement et du développement ${ }^{2}-$, il est presque aussi évident que la phase d'appropriation qui s'en est suivie a davantage fait place à des démarches compétitives, menant à des interprétations pour le moins divergentes du développement durable... Compétition dans laquelle chacun essaie d'imposer la définition du terme la plus compatible avec ses propres intérêts.

Dans ce processus de traduction ${ }^{3}$, il semble que les outils d'évaluation soient amenés à jouer un rôle central : en transformant un concept souvent qualifié de «mou » sous la forme de chiffres (indicateurs, critères), ils s'avèrent en effet de redoutables moyens d'appropriation et de " rigidification ». Pour le chercheur un tant soit peu curieux, la conséquence intéressante de ce phénomène est que, chaque outil d'évaluation devenant le reflet d'une vision spécifique du développement durable, il est alors possible d'utiliser ces instruments pour essayer de définir le contenu substantif que chaque type d'acteur tend à donner à ce terme. En bref, le pouvoir d'appropriation des outils d'évaluation du développement durable est tel que nous pourrions résumer la situation en une phrase simple: «Dis-moi comment tu évalues, et je te dirai quelle est ta vision du développement durable. »

Ce jeu de puzzle devient particulièrement captivant lorsqu'on s'attache au cas particulier des acteurs publics locaux. Chargés, selon la célèbre formule, de «penser

\footnotetext{
1 Voir notamment Boutaud (2005), dont cet article est tiré (travail de recherche mené à l'École nationale supérieure des mines de Saint-Étienne, en partenariat avec l'ADEME, Rhônalpénergie-Environnement et la communauté urbaine de Lyon).

${ }^{2}$ L'intitulé même des organismes (Commission mondiale sur l'environnement et le développement, dite commission Brundtland) et des grands événements internationaux (Conférence des Nations unies sur l'environnement et le développement, dite Sommet de la Terre) qui ont amené à la formulation du concept de développement durable suffit d'ailleurs à rappeler à quel point la question de la conciliation entre environnement et développement fut au cœur de ces négociations.

${ }^{3}$ Le terme de traduction fait référence ici à la définition qui en est donnée en sociologie des sciences, c'est-à-dire la manière dont des acteurs s'insèrent dans un tissu de relations sociales complexes, afin de progressivement imposer leurs points de vue et leurs intérêts (Callon, 1986). Yannick Rumpala (1999) a donné une interprétation intéressante de cette approche de la traduction appliquée au développement durable.
}

global et d'agir local », ces derniers se trouvent en effet au cœur du mécanisme de mise en œuvre du développement durable. À l'heure où celui-ci est sur le point de devenir un mot d'ordre récurrent dans la formulation des politiques locales, il semble donc bien venu de se pencher précisément sur le contenu et la forme que les acteurs locaux s'accordent à lui donner.

Ainsi, après avoir brièvement présenté les outils d'évaluation sur lesquels nous avons porté notre attention, nous essaierons d'en décortiquer le contenu afin d'en tirer des conclusions concernant la «forme » du développement durable qui semble être privilégiée par les acteurs publics au niveau local, en France et en Suisse romande. Cela nous permettra notamment d'ouvrir le débat quant à la pertinence de cette «forme » eu égard à la question, désormais classique, de la fractalité du développement durable (question que nous pourrions résumer de la manière suivante : Convient-il ou non d'adopter une forme similaire du développement durable quel que soit le positionnement des territoires locaux dans la problématique globale?).

\section{Un objet d'étude un peu particulier : les outils de questionnement et d'analyse des politiques et des projets en matière de développement durable}

L'organisation des collectivités locales au sein d'un réseau mondialisé $\left(\mathrm{l}^{\prime} \mathrm{ICLEI}{ }^{4}\right)$ avait permis dès le premier Sommet de la Terre, à Rio de Janeiro, de clairement souligner le rôle fondamental des acteurs publics locaux dans le processus de mise en œuvre du développement durable $^{5}$. «Penser global, agir local» : la formule paraissait en effet séduisante. Néanmoins, force fut de constater, lors du second Sommet de la Terre, à Johannesburg, que les objectifs proposés à Rio étaient loin d'avoir été respectés en la matière ${ }^{6}$. Et pour cause, puisque la proposition faite aux collectivités par les Nations unies ne

\footnotetext{
${ }^{4}$ International Council for Local Environmental Initiatives (www.iclei.org) : organisme créé en 1990 suite au Congrès mondial des collectivités locales pour un avenir durable et dont l'une des premières tâches consista à proposer d'inclure les collectivités locales dans les préoccupations de l'Agenda 21 de Rio.

${ }^{5}$ C'est notamment ce que rappelait en 1992 le chapitre 28 de l'Agenda 21 des Nations unies, en invitant les collectivités locales à mettre en place, d'ici à 1996, des stratégies de développement durable à l'échelle de leur territoire : « [.. ] la plupart des collectivités locales devront avoir entrepris un processus de consultation auprès de leurs populations et être parvenues à un consensus en ce qui concerne un Agenda 21 local pour la collectivité » (Nations unies, 1992).

${ }^{6}$ Le bilan des Agendas 21 locaux réalisé par l'ICLEI en 2002 semblait, de son côté, démontrer que nous étions alors loin de l'objectif fixé en 1992 (International Council for Local Environmental Initiatives, 2002).
} 
pouvait légitimement engager que les signataires de ces textes internationaux, à savoir non pas les collectivités elles-mêmes... mais les États. Dans ce contexte, on ne s'étonnera guère que, d'un pays à l'autre, les taux très variables d'engagement des collectivités dans des stratégies de développement durable s'expliquent avant tout par le volontarisme (ou le défaut de volontarisme) du maillon central que sont les États. La France, pays qui figure en queue de peloton des nations européennes en termes de réalisation d'Agendas 21 locaux, en est un bon exemple: il aura fallu attendre 2003 pour qu'une stratégie nationale du développement durable soit enfin adoptée et que des engagements explicites soient pris en la matière ${ }^{7}$.

Mais ce contexte national assez timoré n'a heureusement pas empêché un certain nombre d'acteurs publics de progressivement s'engager sur la voie du développement durable, en élaborant notamment, chacun de leur côté, tout un attirail d'outils et de processus visant à intégrer le développement durable dans leurs politiques respectives. Parmi ces instruments, les outils de questionnement et d'analyse des politiques et projets en matière de développement durable (OQADD) figurent en bonne place.

\section{Les OQADD : définition générale}

Souvent appelés "grilles développement durable», les OQADD sont des objets très hétéroclites apparus, pour la plupart, de manière assez anarchique dans le paysage administratif et politique francophone. Certains furent créés pour évaluer des projets dans le cadre d'un appel d'offres (ex. : appels à projet du ministère de l'Environnement français sur les outils et les démarches en vue de la réalisation d'Agendas 21 locaux), d'autres, pour aider les porteurs de projet d'un territoire à mieux prendre en compte les critères du développement durable (ex. : le $\mathrm{SGAR}^{8}$ Franche-Comté, dans le cadre du projet de développement durable entre Saône et Rhin), d'autres encore, pour rendre le concept de développement durable plus facilement assimilable par les personnels administratifs (ex : conseil régional du Nord-Pas-de-Calais).

Développés sur le terrain par des acteurs publics, des associations ou éventuellement des bureaux d'études, les OQADD ainsi conçus se réclament à la fois de l'évaluation des politiques et de l'analyse multicritère, tout en étant bien souvent à mille lieues des canons théoriques développés par ces différentes approches. De manière assez synthétique, nous considérerons donc que peuvent

\footnotetext{
${ }^{7}$ Notons, à ce propos, que la Stratégie nationale du développement durable française inclut un volet spécifiquement dédié aux acteurs locaux et fixe un objectif de 500 Agendas 21 locaux pour l'horizon 2008. Mais cet objectif n'engage là encore que l'État (Comité interministériel du développement durable, 2003).

8 Secrétariat général pour les affaires régionales.
}

être regroupés sous cet intitulé très vague d'OQADD des dispositifs :

- dont la finalité consiste à évaluer, à analyser ou, plus simplement, à questionner des politiques ou des projets (dont l'état d'avancement est variable) par rapport aux exigences du développement durable;

- et dont la forme est le plus souvent celle d'une grille de critères, organisés et hiérarchisés en arborescence, et formulés sous forme de questions dont les résultats peuvent être (mais ne sont pas nécessairement) agrégés et pondérés entre eux.

\section{Les OQADD : finalité. . .}

Un travail de recensement, mené en France et en Suisse romande dans le courant de l'année 2004, nous avait permis d'identifier à cette époque 33 démarches en cours et 26 OQADD effectivement réalisés et opérationnels (Boutaud, 2005). Concernant tout d'abord leur finalité, il convient de constater que ces outils ne sont pas, a priori, destinés systématiquement à une évaluation strictement entendue comme «l'appréciation a posteriori des effets réels des décisions publiques » (Gault et Galmiche, 2000). La finalité des OQADD pourrait néanmoins correspondre à une définition plus large de l'évaluation, comme, par exemple, celle donnée par Patrick Viveret (1989) lorsqu'il affirme qu' « évaluer une politique, c'est former un jugement sur sa valeur ». Mais, quelle que soit la définition retenue de l'évaluation, force est en tout cas de constater que très peu de ces outils se réfèrent explicitement au terme d'évaluation dans leur intitulé. La grande majorité des OQADD préfèrent renvoyer à des notions plus vagues et, surtout, plus consensuelles : grille de lecture, questionnement, analyse...

De fait, ces termes semblent assez bien représenter la finalité de la plupart des OQADD. À part quelques cas spécifiques d'outils dédiés à la sélection de projets ou au diagnostic de politiques, la majorité des OQADD ont comme objet l'aide à la décision. En effet, le développement durable est le plus souvent appréhendé comme un "supracritère" auquel les utilisateurs des OQADD confrontent leurs projets ou leurs politiques, bien que ceux-ci ne correspondent qu'en partie au système de valeurs du développement durable. En termes de finalités, on pourrait donc classer ces OQADD dans une catégorie un peu à part : celle de «l'aide à la prise en compte d'un facteur exogène » (le développement durable, en l'occurrence) dans les projets.

\section{... et forme}

Cette tendance en termes de finalités a bien entendu des conséquences sur la forme que prennent ces dispositifs. Les OQADD se présentent généralement comme 


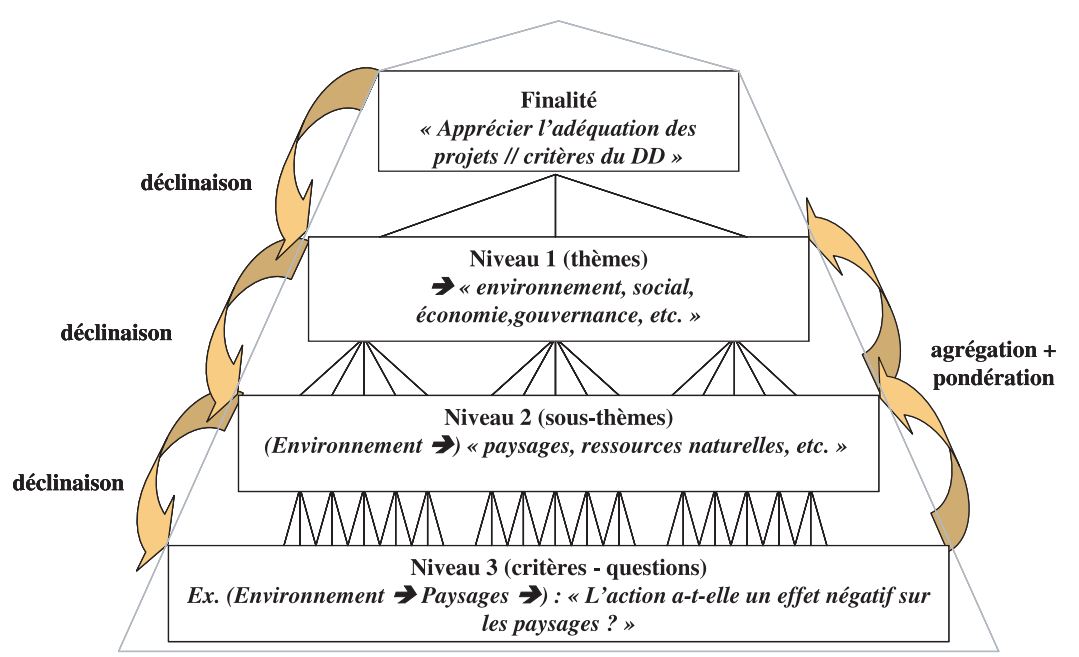

Fig. 1. Arborescence type d'un OQADD : le développement durable et sa déclinaison en critères et questions explicites.

des grilles d'analyse multicritère organisées en arborescence et déclinant en plusieurs niveaux les différentes dimensions du développement durable (économie, environnement, social, gouvernance, autres principes, etc.). Ces thèmes sont le plus souvent énoncés sous forme de critères, puis de questions ouvertes ou fermées qui interrogent directement la politique ou le projet identifié (Fig. 1). Mais, à part là encore quelques cas, les OQADD sont pour la plupart des outils d'analyse multicritère assez incomplets qui se cantonnent à une position de questionnement ou d'interrogation, sans aller bien au-delà de cette mise en perspective un peu globale. En particulier, les questions ne donnent pas systématiquement lieu à une notation (niveau de réponse), et il n'est quasiment jamais fait référence à un quelconque système d'agrégation qui permettrait d'obtenir un résultat plus synthétique et lisible (par exemple : le projet répond à $x \%$ aux critères du développement durable).

Malgré leur très grande hétérogénéité, on retiendra surtout que le principal point commun des OQADD réside dans le fait qu'ils obligent au minimum leurs concepteurs à organiser et à hiérarchiser un certain nombre de critères, et donc à exprimer de manière très explicite ce qu'ils entendent par développement durable... Ce qui est précisément le point qui nous intéresse dans le cadre de cet article.

\section{La vision du développement durable adoptée par les acteurs publics locaux : une approche largement consensuelle. . . et fractale}

Dans un article portant sur l'évaluation monétaire du développement durable, Sandrine Rousseau-de Vetter (2000) émet l'hypothèse intéressante selon laquelle, à chaque type d'approche évaluative, correspondrait une forme de durabilité ( «très faible » à « très forte ${ }^{9}$ »). Ainsi, l'évaluation contingente correspondrait à la vision d'une durabilité très faible, typique des approches néoclassiques "éconocentrées », tandis que l'analyse multicritère concorderait davantage avec une approche de la durabilité forte, plus «écolocentrée ». Ce dernier point porte néanmoins à discussion : l'évaluation multicritère semble aussi bien pouvoir refléter une approche faible qu'une approche forte. Le cas de certains indicateurs agrégés, en particulier ceux développés respectivement par les Amis de la Terre (E-HDI : Environmental Human Development Index) et le Forum économique mondial de Davos (ESI : Environmental Sustainability Index) (Fig. 2), semble fournir une bonne illustration de cette capacité des approches multicritères à s'émanciper d'un courant de pensée, pour refléter avant tout la vision du développement durable des concepteurs ou des commanditaires de l'outil d'évaluation en question. Les problèmes fondamentaux posés par les étapes de choix des critères et surtout de pondération et d'agrégation font qu'à chaque moment, l'outil d'analyse peut être orienté vers une approche de la durabilité faible, forte. . . ou encore consensuelle.

Reprenant les différentes phases de traduction du concept (choix des critères, pondération-agrégation), nous allons voir que c'est précisément une approche consensuelle du développement durable, typique des acteurs publics, que les OQADD tendent à refléter.

\section{Précisions méthodologiques}

Afin de déterminer le contenu «idéologique » des OQADD, il nous a fallu décortiquer ces outils en procédant en deux étapes :

- identification et classification des critères de chaque OQADD selon plusieurs catégories sémantiques,

\footnotetext{
9 Selon la typologie proposée notamment par Turner (1993) ou encore Dobson (1996).
} 


\begin{tabular}{|l|c|c|}
\hline & ESI & E-HDI \\
\hline Norvège & $\mathbf{1}$ & $\mathbf{1 6}$ \\
\hline Finlande & $\mathbf{2}$ & $\mathbf{1 7}$ \\
\hline Canada & $\mathbf{3}$ & $\mathbf{6}$ \\
\hline Islande & $\mathbf{4}$ & $\mathbf{2 0}$ \\
\hline Suède & $\mathbf{5}$ & $\mathbf{1 2}$ \\
\hline Suisse & $\mathbf{6}$ & $\mathbf{2}$ \\
\hline Australie & $\mathbf{7}$ & $\mathbf{1 0}$ \\
\hline Danemark & $\mathbf{8}$ & $\mathbf{1 9}$ \\
\hline Etats-Unis & $\mathbf{9}$ & $\mathbf{1 4}$ \\
\hline Pays-Bas & $\mathbf{1 0}$ & $\mathbf{1 8}$ \\
\hline$\ldots$ & $\mathbf{1 1}$ & $\mathbf{1 1}$ \\
\hline Algérie & $\mathbf{1 2}$ & $\mathbf{1 5}$ \\
\hline Singapour & $\mathbf{1 3}$ & $\mathbf{1 3}$ \\
\hline Sénégal & $\mathbf{1 4}$ & $\mathbf{9}$ \\
\hline Malawi & $\mathbf{1 5}$ & $\mathbf{3}$ \\
\hline Vietnam & $\mathbf{1 6}$ & $\mathbf{4}$ \\
\hline Iran & $\mathbf{1 0}$ & $\mathbf{7}$ \\
\hline Nigeria & $\mathbf{1 0}$ \\
\hline Ouganda & & \\
\hline Bangladesh & & \\
\hline Madagascar & & \\
\hline
\end{tabular}

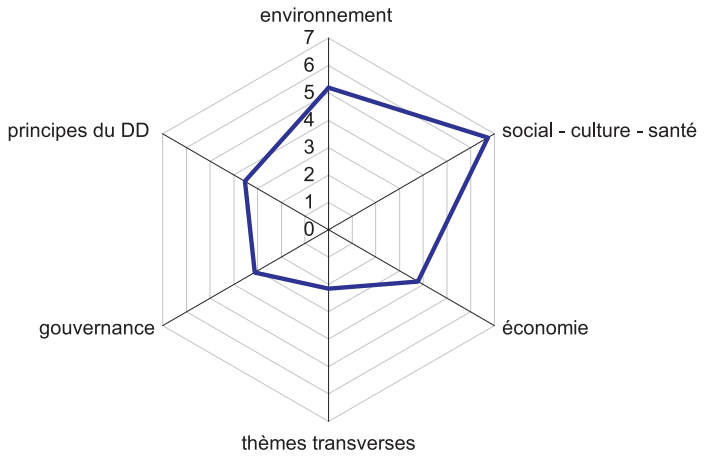

Fig. 3. Répartition des critères (nombre moyen de critères cités) par catégorie sémantique dans les 26 OQADD étudiés.

- catégorie 3 : économie (rentabilité, emplois, tissu économique...)

- catégorie 4 : thèmes transverses, champs croisés (transversalité, internalisation...)

- catégorie 5 : gouvernance (information, concertation, participation)

- catégorie 6 : principes du développement durable (précaution, long terme, global/local...).

\section{Une répartition équilibrée des critères entre les trois «sphères » du développement durable}

La figure 3 dresse le portrait-robot des OQADD étudiés : il s'agit de la répartition moyenne des critères identifiés en fonction de leur appartenance à chacune des catégories sémantiques susmentionnées. Qu'en conclure?

Fig. 2. Durabilité faible vs durabilité forte : comparaison classements d'un échantillon de vingt nations en matière de développement durable selon deux indicateurs différents : 1 'ESI (World Economic Forum of Davos) et l'E-HDI (Friends of the Earth UK).

Source : ce tableau comparatif est issu d'un communiqué de l'ONG Friends of the Earth UK paru en avril 2001. Il prend en compte uniquement les pays classés aux dix premiers et aux dix derniers rangs en matière $\mathrm{d}^{\prime} \mathrm{ESI}$.

afin de déterminer les tendances thématiques dominantes ;

- analyse des modes d'agrégation et de pondération des critères, afin de déterminer les préférences mises en œuvre dans chaque OQADD.

Notre analyse s'est portée en particulier sur la totalité des critères du dernier niveau de chacune des 26 grilles multicritères, que nous avons classés en plusieurs catégories sémantiques, selon l'appartenance des différents critères aux thématiques suivantes :

- catégorie 1 : environnement (ressources, milieu et cadre de vie...)

- catégorie 2 : social - culture - santé (solidarité, équité, santé, culture...)

\section{Confirmation. . .}

Tout d'abord, quelques éléments chiffrés viennent effectivement confirmer l'impression d'équilibre dans la répartition des critères pour l'ensemble des OQADD. Sur les 684 critères relevés, 19,7\% sont relatifs à des questions d'environnement, 25,6 \% à des thématiques sociales et culturelles (incluant également les thématiques de santé et de pédagogie), et 14,3\% à des préoccupations purement économiques. Tous les OQADD font référence de manière explicite à au moins un critère dans chacune des sphères du triptyque. Les critères transversaux (56, soit $8,2 \%$ ) viennent rééquilibrer légèrement cette répartition avantageuse en faveur des thématiques socioculturelles, puisqu'ils concernent majoritairement l'intégration des questions économiques et environnementales.

\section{...et nuances}

Une lecture au cas par cas met pourtant en évidence une certaine hétérogénéité dans la répartition des catégories de critères entre les différents outils. La moitié 
des OQADD présente la caractéristique d'avoir le plus grand nombre de critères au sein de la catégorie 2 (critères socioculturels). Quelques-uns (6) ont, au contraire, davantage de critères dans le domaine environnemental et d'autres, plus rares (2), dans le secteur économique. À part 3 OQADD, la répartition entre critères reste néanmoins assez équilibrée entre les différentes sphères du développement durable. Enfin, trois outils se démarquent des autres en ce qu'ils semblent mettre l'accent plus sur les «principes » du développement durable que sur ses «dimensions ».

Dans tous les cas, on ne peut pas vraiment dire que le développement durable nous apparaisse, à travers ces critères (ou du fait de leur répartition), comme un concept relevant majoritairement de l'une des trois sphères (écologique, sociale ou environnementale). Une impression de «tiédeur» que le refus quasi général d'agrégation et de pondération des critères vient encore largement renforcer. .

\section{Agrégation et pondération des critères : des questions qui fâchent}

La grande majorité des OQADD ne se soucient pas vraiment de ces questions d'agrégation et de pondération des critères, préférant en rester au stade de la formulation de questions relatives à chaque critère, sans agrégation des réponses a posteriori. Les rares OQADD qui agrègent pour partie des résultats n'opèrent pas de pondération des critères. Ce qui signifie que l'agrégation consiste en une moyenne arithmétique des résultats obtenus pour chaque question correspondant à un critère. Pour cette raison, la plupart des OQADD qui ont fait le choix d'une agrégation partielle des données comprennent un nombre équivalent de critères au sein de chaque thématique, afin de ne pas donner plus d'importance à une dimension par rapport aux autres.

Le lecteur l'aura compris, on touche là une question centrale : dans la définition du développement durable que l'on décide d'adopter, faut-il donner davantage d'importance à l'une des dimensions du triptyque environnement-social-économie? Si, pour les Amis de la Terre ou le Forum économique de Davos (Fig. 2), la réponse à cette question est évidemment positive, l'idée même de pouvoir donner davantage d'importance à l'une de ces trois dimensions semble par contre totalement hérétique aux yeux de la plupart des acteurs publics. Le rapporteur d'un atelier de travail portant sur le sujet des grilles d'évaluation du développement durable illustre assez bien cette position : «Pour certains [membres du groupe de travail], une pondération devrait être faite entre des facteurs sociaux, environnementaux et économiques. Pour d'autres, ceci est hautement dangereux et irait dans le sens contraire du principe du développement durable qui veut justement mettre au même niveau les trois axes du développement » (Bellini, 2002). Une autre personne ayant mis au point un OQADD est, quant à elle, encore plus claire sur le sujet : «Le développement durable postulant de prendre en compte de manière égale l'économie, le social et l'environnement, il s'agit de faire en sorte que la même importance soit donnée aux trois domaines dans les résultats » (Münster, 2002). Et c'est bien cette dernière solution qui semble avoir été retenue pour la grande majorité des OQADD étudiés.

Ainsi, tant par leur contenu sémantique (répartition des critères) que par leur mode d'organisation hiérarchique (agrégation-pondération des critères), les OQADD mis au point par les acteurs publics locaux semblent refléter une vision très consensuelle du développement durable - approche qui se caractérise en particulier par le traitement sur un strict pied d'égalité des questions environnementales, sociales et économiques. Or, si cette vision du développement durable semble aller de soi pour la grande majorité des acteurs publics locaux, sa pertinence interroge dès lors que l'on reformule la question dans une perspective plus globale.

\section{Penser global, agir local... À propos de la (non-)pertinence d'une approche fractale du développement durable au niveau local : quelques pistes de réflexion}

Comme nous l'avons noté plus haut, le concept de développement durable est né dans un contexte de tentative de réconciliation entre environnement et développement au niveau mondial, aboutissant finalement à un point d'entente entre ces exigences via la notion de solidarité intergénérationnelle. C'est donc à un consensus sur le nécessaire élargissement de la notion d'intérêt général dans le temps (prise en compte du long terme et des générations futures) que la réflexion internationale sur le développement durable a fini par aboutir. Mais, en se territorialisant, le développement durable renvoie également à une dimension spatiale : à la nécessaire solidarité vis-à-vis des générations futures, vient s'ajouter un second principe de solidarité vis-à-vis des autres territoires, du local (territoires adjacents) au global (reste de la planète). Or, en la matière, rien ne prouve que les intérêts locaux et globaux soient systématiquement convergents.

\section{Développement durable et territoires : le « dilemme du glocal »...}

Cette problématique du conflit d'intérêt entre local et global est ainsi progressivement apparue comme une question centrale, au cours des dernières années, parmi 
les théoriciens de l'approche territoriale du développement durable. Elle est, par exemple, largement abordée par Jacques Theys et Cyria Emelianoff (2001), qui se demandent si une ville durable doit « $d$ 'abord contribuer à la solution des grands problèmes mondiaux - au prix éventuel de sa croissance - ou plutôt s'assurer de la viabilité à long terme de son propre développement ». Car l'invitation à une action locale basée sur une pensée globale, si elle est généreuse, a également toutes les chances d'être vaine tant, comme le fait remarquer Olivier Godard (1996), la soutenabilité du développement global « dépend à la fois de la viabilité interne $\mathrm{d}^{\prime}$ un système territorial et de sa viabilité externe (effet sur les systèmes territoriaux supérieurs) ». Wackernagel et Rees (1999), de leur côté, parlent de durabilité interne et durabilité externe, tandis que Bertrand Zuindeau (2000) confirme cette ambiguité et cite notamment les travaux de David Pearce et de ses collègues, qui introduisent la notion de durabilité importée et de non-durabilité exportée : chaque territoire tendrait naturellement à résoudre ses problèmes internes en les délocalisant vers l'extérieur, ce qui tend à résoudre le problème local sans résoudre le problème global (Pearce et al., 1989; voir également Nijkamp et Vreeker, 2000 ; Gibbs, 1997).

Cette tentation des territoires de favoriser leur durabilité interne en faisant porter sur les autres leurs problèmes est qualifiée par Christian Brodhag (2003) de «stratégie du passager clandestin ». En effet, la possibilité d'un dilemme du prisonnier (que nous baptiserions volontiers «dilemme du glocal») apparaît entre les villes ou les territoires qui joueraient complètement le jeu du développement durable et les autres : les territoires engagés dans une démarche volontaire risqueraient de se pénaliser en essayant de résoudre des problèmes globaux que $\mathrm{d}$ 'autres collectivités ne prendraient pas en compte.

La question, on le constate, n'est pas mince. Et, même si de nombreux auteurs (Camagni et al. 1998; Theys et Emelianoff, 2001) s'avèrent plutôt optimistes, soulignant que les synergies entre ces intérêts globaux et locaux permettent parfois de surmonter ce «dilemme du glocal ${ }^{10}$, ces cas de double dividende sont loin d'être systématiques.

\section{... et la question de la fractalité du développement durable}

Ce «dilemme du glocal » interroge donc sur la forme que doit recouvrir le développement durable au niveau local. Ainsi, à une thèse selon laquelle, "pour que le développement planétaire soit soutenable, il suffit que

\footnotetext{
10 Par exemple, la lutte contre les problèmes locaux de congestion du trafic et de pollution atmosphérique concorde avec les intérêts globaux qu'il y a à lutter contre le changement climatique.
}

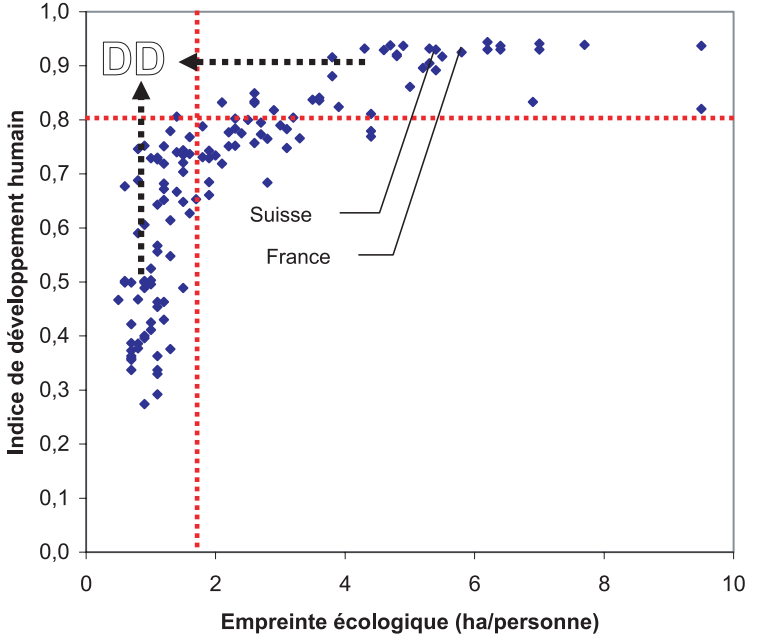

Fig. 4. Un même objectif, des chemins différents : indice de développement humain (IDH) et empreinte écologique de 130 nations du monde pour l'année 2000 (d'aprés Boutaud et al., 2004). Le seuil de 0,800 IDH représente un niveau élevé de développement humain selon l'ONU ; le seuil 1,9 ha/hab. est la surface de sol bioproductif disponible par habitant de la Terre, ou seuil de durabilité écologique; les flèches symbolisent les chemins radicalement différents qu'il convient de suivre pour atteindre l'objectif d'un développement humain à la fois élevé et écologiquement durable, selon le positionnement qu'un pays ou un territoire occupe au niveau mondial.

le développement de chaque espace local ou ensemble urbain soit lui-même viable », Olivier Godard (1996) oppose une antithèse selon laquelle «la viabilité locale peut n'être pas suffisante; la poursuite de ce type de viabilité peut même représenter l'une des causes importantes de la non-soutenabilité du développement aux échelles territoriales supérieures ». Godard en conclut que le développement durable est peu fractal dans la pertinence de ses formes géographiques : il ne saurait recouvrir la même réalité, les mêmes priorités et donc les mêmes formes dans toutes les régions.

Cette question, longtemps traitée de manière assez théorique, trouve aujourd'hui une illustration assez intéressante via l'utilisation de certains indicateurs que nous pourrions précisément qualifier de «durabilité externe » (ou durabilité globale), telle l'empreinte écologique. Le croisement des résultats de cet indice (qui mesure la pression exercée par une société sur l'ensemble des ressources écologiques mondiales) avec ceux de l'indice de développement humain (IDH) de 130 nations du monde (Fig. 4) met clairement en évidence les déséquilibres de situation entre territoires du «Nord» et du «Sud».

Partant de là, si nous acceptons le constat selon lequel « les pays du Nord ont autant d'efforts à fournir pour devenir écologiquement durables que les pays du Sud pour devenir socio-économiquement développés » (Boutaud, 2002), alors force est de nous poser une série de questions cruciales quant à la fractalité du développement 


\section{Encadré. Pour une nouvelle «géographie» du développement durable?}

Imaginons que nous demandions à un Perpignanais et à un Strasbourgeois de se rendre à Brest en leur précisant simplement que cette ville se trouve au nord-ouest de la France. Cela ne veut pas dire pour autant que les deux (pourtant bien français) devront suivre la direction du nord-ouest. L'un devra aller plus à l'ouest qu'au nord et l'autre, inversement.

La boussole à deux ou trois dimensions du développement durable ne nous mènera nulle part si nous ne sommes pas capables avant tout de nous situer sur la surface du globe (ce que permet, par exemple, le croisement IDH-empreinte écologique : Fig. 4, en montrant clairement que les chemins à suivre sont différents pour les pays riches et pour les " pauvres »)... Et c'est ce point de départ qui doit ensuite nous permettre de nous orienter, non pas plus au Sud ou plus au Nord, mais en prenant en compte au niveau local, de manière plus ou moins prioritaire, une dimension du développement durable par rapport à une autre.

durable : Est-il légitime que les territoires du Nord et $\mathrm{du}$ Sud adoptent une approche identique (fractale) et consensuelle (équilibrée) du développement durable? Le positionnement de chaque territoire dans la problématique mondiale $\mathrm{n}^{\prime}$ impose-t-il pas aux territoires du Nord et du Sud d'adopter des objectifs différents, et donc des approches elles aussi radicalement distinctes (et nonfractales) du développement durable? (Encadré.)

Pour être encore plus clairs, nous pourrions finalement formuler la question de cette manière : Le même consensus mou, qui consiste à affirmer la nécessité d'adopter en tous lieux une approche équilibrée entre les «trois piliers » du développement durable, est-il susceptible de pallier les graves déséquilibres mondiaux qui caractérisent précisément la problématique du développement durable?

\section{Conclusion}

L'analyse des OQADD développés au niveau local nous révèle une vision du développement durable éminemment consensuelle. L'idée qui consiste à devoir traiter sur un strict pied d'égalité les questions environnementales, sociales et économiques tend ainsi à progressivement s'imposer parmi les acteurs publics comme un élément de définition incontournable du développement durable. Or, cette approche équilibrée pose pour le moins des questions lorsqu'on resitue ce débat local dans son contexte international.

Et il semble bien que nous soyons là confrontés à un problème de fond, un nouveau paradoxe du développement durable. Alors que la problématique mondiale paraît se cristalliser autour de la question préoccupante de l'accroissement des déséquilibres écologiques et sociaux, la réponse pour le moins paradoxale apportée au niveau local consisterait en l'adoption d'une approche équilibrée, répétée à l'identique partout autour de la planète. . et dont on imagine finalement assez mal comment elle pourrait faire autre chose qu'entretenir ces déséquilibres ${ }^{11}$.

Au final, l'introduction du «penser global » dans $l^{\prime}$ «agir local» nous confronte ainsi inévitablement à la

\footnotetext{
11 À moins, bien entendu, qu'elle ne serve à légitimer ces mêmes déséquilibres?
}

question de la légitimité des systèmes d'action publique locaux - légitimité jusque-là basée (dans les pays démocratiques du moins) sur une vision finalement bien restreinte de l'intérêt général. Une définition que le développement durable fait éclater de toutes parts : dans le temps, d'abord (en introduisant la nécessaire prise en compte des intérêts des générations futures), mais également dans l'espace (en posant la question des intérêts des autres territoires et du reste du monde). On le constate aisément, derrière la formulation apparemment anodine du slogan «penser global, agir local », les défis en matière d'organisation démocratique des prises de décision sont de taille...

\section{Remerciements}

Les auteurs tiennent à remercier les membres du comité de lecture, et en particulier les relecteurs du présent article, pour la pertinence de leurs remarques.

\section{Références}

Bellini, E., 2002. Résumé de l'atelier 2 : «Qu'est-ce qu'un projet durable dans une commune ou dans un canton?» ou "Comment évaluer des projets selon des critères de DD? », in ARE (Ed.), Forum du développement durable $n^{\circ} 4$. Annexes, Berne, ARE (téléchargeable sur Internet, mars 2005 : http://www.are.admin.ch/imperia/md/content/are/ nachhaltigeentwicklung/deutsch/forumne/11.pdf).

Boutaud, A., 2002. Développement durable : quelques vérités embarrassantes, Économie et Humanisme, 363, 4-6 (repris dans Boutaud, A., 2003. Développement durable : à la recherche des bons indicateurs, Problèmes économiques, 2800, 1-3).

Boutaud A., 2005. Le Développement durable: penser le changement ou changer le pansement? Bilan et analyse des outils d'évaluation des politiques publiques locales en matière de développement durable en France: de l'émergence d'un changement dans les modes de faire au défi d'un changement dans les modes de penser. Thèse de doctorat en sciences de la Terre et de l'environnement, École des mines de Saint-Étienne / Université Jean Monnet, Saint-Étienne.

Boutaud, A., Brodhag, C., Gondran, N., 2004. Lorsque le développement perd le Nord! Courbes de Kuznets Environnementales : 1'apport des indicateurs alternatifs de type empreinte écologique dans la réflexion sur le développement durable, in OIF (Ed.), Développement durable : leçons 
et perspectives, Actes du colloque scientifique de Ouagadougou préparatoire au $10^{e}$ Sommet de la francophonie, Paris, OIF, 27-34 (téléchargeable sur Internet, mars 2005 :

http://www.francophonie-durable.org/ documents/ colloque-ouaga-a3-boutaud.pdf).

Brodhag, C., 2003. Genèse du concept de développement durable : dimensions éthiques, théoriques et pratiques, in Da Cunha, A., Ruegg, J. (Eds), Développement durable et aménagement du territoire, Lausanne, Presses polytechniques et universitaires romandes, 29-45.

Callon, M., 1986. Éléments pour une sociologie de la traduction. La domestication des coquilles Saint-Jacques et des marinspêcheurs dans la baie de Saint-Brieuc, L'Année sociologique, 36, 169-208.

Camagni, R., Capello, R., Nijkamp, P., 1998. Towards sustainability policy : an economy-environment technology nexus, Ecological Economics, 24, 103-118.

Comité interministériel du développement durable, 2003. Stratégie nationale du développement durable, Paris, CIDD/MEDD.

Dobson, A., 1996. A typology of environmental sustainabilities, Environmental Policies, 5, 3, 401-428.

Gault, M., Galmiche, C., 2000. L'Évaluation des politiques publiques urbaines, Paris, Ministère de l'Équipement, des Transports et du Logement, Direction générale de l'urbanisme, de l'habitat et de la construction.

Godard, O., 1996. Le développement durable et le devenir des villes ; bonnes intentions et fausses bonnes idées, Futuribles, 209, 29-35.

Gibbs, D., 1997. Urban sustainability and economic development in the United Kingdom: exploring the contradictions, Cities, 14, 4, 203-209.

International Council for Local Environmental Initiatives, 2002. Réponses des gouvernements locaux à Action 21 : rapport sommaire de l'enquête régionale sur les programmes Action 21 locaux, Toronto, ICLEI.

Münster, M., 2002. Quelques pistes de réflexions essentielles à la mise en place de grilles d'évaluation de projets d'après les critères du développement durable, in ARE (Ed.) Forum du développement durable $n^{\circ} 4$. Annexes, Berne, ARE (téléchargeable sur Internet, mars 2005 :

http://www.are.admin.ch/imperia/md/content/are/ nachhaltigeentwicklung/deutsch/forumne/11.pdf).

Nations unies, 1992. Agenda 21, New-York, Nations unies (téléchargeable sur Internet, avril 2005 :

http://www.un.org/esa/sustdev/documents/agenda21/ french/action0.htm).

Nijkamp, P., Vreeker, R., 2000. Sustainability assessment of development scenarios : methodology and application to Thailand, Ecological Economics, 33, 7-27.

Pearce, D., Markandya, A., Barbier, E.B., 1989. Blueprint For a Green Economy, London, Earthscan.

Rousseau-de Vetter, S., 2000. Pourquoi et comment l'évaluation monétaire peut-elle servir localement la problématique du développement durable?, in Zuindeau, B. (Ed.), Développement durable et territoires, Villeneuve-d'Ascq, Presses universitaires du Septentrion, 108-141.

Rumpala, Y., 1999. Questions, écologiques, réponses économiques : les changements dans la régulation publique des problèmes d'environnement au tournant des années 1980 et 1990, une analyse intersectorielle. Thèse de doctorat en science politique, Institut d'études politiques, Paris.

Theys, J., Emelianoff, C., 2001. Les contradictions de la ville durable, Le Débat, 113, 122-135.

Turner, R. K., 1993. Sustainable Environmental Economics and Management: Principles and Practice, London, Belhaven.

Viveret, P., 1989. L'Évaluation des politiques et des actions publiques: propositions en vue de l'évaluation du Revenu Minimum d'Insertion. Rapport au Premier ministre, Paris, La Documentation française.

Wackernagel, M., Rees, W., 1999. Notre empreinte écologique, Québec, Écosociété.

Zuindeau, B., 2000. La durabilité : essai de positionnement épistémologique du concept, in Zuindeau, B. (Ed.), Développement durable et territoires, Villeneuve-d'Ascq, Presses universitaires du Septentrion, 27-69.

Received 21 avril 2005. Accepté 5 janvier 2006.

To access this journal online: www.edpsciences.org 